\title{
Cancer Theranostics: Bridging Conventional and Nano-photodynamic Therapy
}

\author{
Poorani Gananathan ${ }^{1}$ Anirban Chakraborty² Indrani Karunasagar ${ }^{2}$ \\ ${ }^{1}$ Nitte (Deemed to be) University, University Enclave, Deralakatte, \\ Mangalore, Karnataka, India \\ ${ }^{2}$ Nitte University Centre for Science Education \& Research, \\ Nitte (Deemed to be) University, Deralakatte, Mangalore, \\ Karnataka, India

\begin{abstract}
Address for correspondence Poorani Gananathan, PhD, Research Coordinator, Nitte (Deemed to be) University, University Enclave, 6th Floor, Deralakatte, Mangalore 575018, Karnataka, India (e-mail: poorani.g@nitte.edu.in).
\end{abstract}

J Health Allied Sci ${ }^{\mathrm{NU}} 2020 ; 10: 3-8$

\begin{abstract}
Cancer is of various kinds, so are the treatment modalities. Worldwide, cancer is the second leading cause of death, accounting for a whopping 9.6 million deaths in 2018 . Globally, approximately one in six deaths is attributed to cancer. Photodynamic therapy (PDT) is a therapeutic strategy for the treatment of superficial lesions, warts, Barrett's esophagus, premalignant lesions, malignant tumors, and ophthalmic diseases. The literature on PDT is approximately one-third of that in radiation therapy, yet the clinical implementation of PDT in cancer is relatively less. Despite substantial research, the clinical application of photodynamic strategy in cancer therapy is still in its infancy with only a limited number of case studies reported so far. The limitations of the photosensitizer and the shallow depth of penetration of light source are the key technical impediments. However, the use of nanomedicine in PDT can overcome these obstacles. Thus, it is necessary to gain knowledge on how nanomaterials can be merged

Keywords

- cancer

- photodynamic therapy

- nanoparticles

- theranostics with PDT and how it can be utilized in cancer theranostics. In this article, the focus is to understand how PDT works and how it can be utilized in improving the sensitivity of the existing diagnostic and therapeutic techniques. The article also addresses the current challenges for PDT and the future prospects of this technique, particularly in the area of diagnosis and treatment of cancer.
\end{abstract}

\section{Introduction}

Photodynamic therapy (PDT) is a combination therapy that uses photosensitive agents to be absorbed/adsorbed by cells, followed by light source irradiation that produces unstable oxygen radicals or reactive oxygen species (ROS) which mediates cell death ( - Fig. $\mathbf{1}) .^{1}$ The photosensitizing agent is either injected into the bloodstream or applied to the skin superficially, depending on the region of the body being treated. ${ }^{2}$ Once the drug is absorbed by the cancer cells, only the region to be treated is irradiated by a light source of a particular wavelength. The depth of light penetration in the body depends on the incident light's wavelength and intensity. ${ }^{3}$ The photosensitizer excited by photons generates free cytotoxic radicals such as single oxygen and radicals of superoxide. These free radicals selectively destroy the cells bound to the sensitizers. ${ }^{4}$
Further, photosensitizer excitation by an incident photon generates reemission of a fluorescent photon that can be used to visualize tiny tumor deposits, micrometastasis, extent of disease progression, as well as incomplete excision, and residual disease left after radical surgery. ${ }^{5}$ PDT is therefore the method that can be used to detect and destroy micrometastases and residual disease. It also has the ability to be used as a preoperative adjuvant treatment. However, PDT is still regarded as an evolving technique and is not used by oncologists in routine clinical settings.

The origin of the usage of light in medicine and surgery can be traced back to ancient times. Phototherapy started in India, ancient Egypt, and Greece but disappeared for many decades until it was rediscovered at the start of the 10th century by Romans. Sunlight was thought to be the only element of treating illnesses, such as vitiligo, psoriasis, rickets, skin
DOI https://doi.org/

10.1055/s-0040-1709959

ISSN 2582-4287.
Copyright @2020 Nitte University (Deemed to be University)
License terms

()(1) $\Theta \circledast$ 


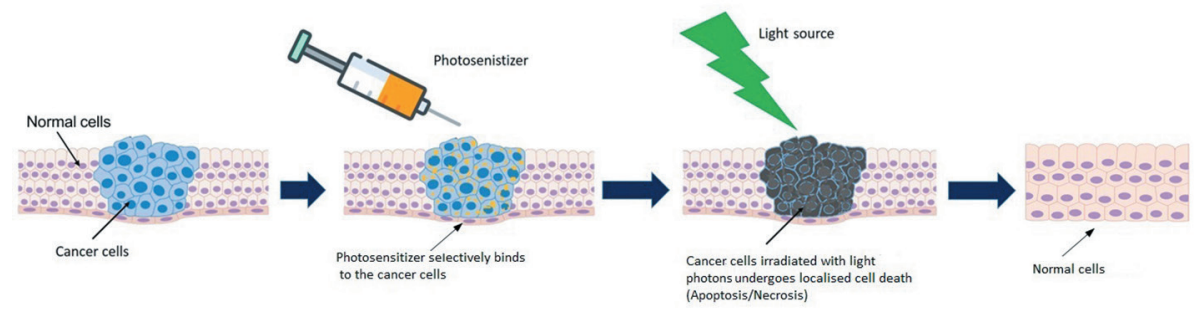

Fig. 1 Mechanism of photodynamic therapy.

cancer, and even psychosis, in those days and this method of therapy (using sunlight) was known as phototherapy or heliotherapy. However, it was only in the 1800s and early 1900 s that phototherapy came into limelight again.-9 In 1903 , Tappenier was the first to use photosensitizer for cancer treatment using topical eosin and white light on skin tumor. ${ }^{10}$ However, this early work did not lead to any important advancements impacting clinical application. The use of a light-absorbing chemical in biological system to trigger photoreactions dates back to 1900 when Raab reported the generation of light-exposed paramecium with an acridine dye. ${ }^{10}$ In 1960, hematoporphyrin derivative (HPD), a mixture of porphyrin, prepared by Swartz, was reported to exhibit tumor fluorescence. However, the systemic studies were done in tumor-bearing animals and later the first clinical report on a patient treated for bladder cancer using HPD was reported. Since then, several clinical studies for treatment of a plethora of tumors employing PDT have been reported by various groups. Shackley et al analyzed the basic principles and applications of PDT and speculated on its likely impact in the near future. In the latter half of the 20th century, the therapeutic use of light in medicine increased with the development of laser and fiber optic technology. ${ }^{10,11}$ PDT harnesses the nonradioactive light energy through a photosensitizing medication with consequent localized tissue necrosis and this method has already undergone clinical trials in some 10,000 patients with malignant, inflammatory, and degenerative circumstances. ${ }^{12}$ Therefore, this is truly an interdisciplinary technique that combines many fields, such as optical physics, engineering, biology, and pharmacology, to cater to

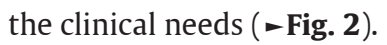

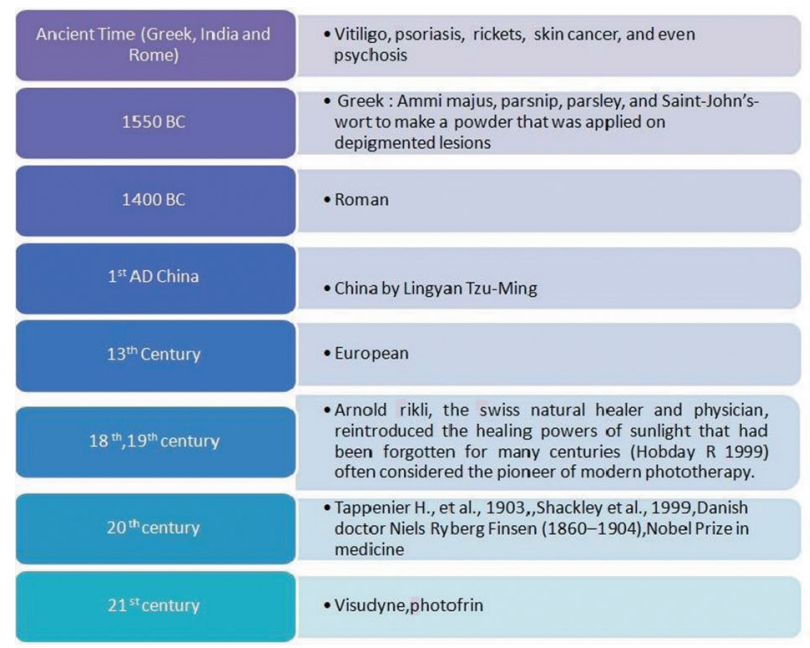

Fig. 2 History of photodynamic therapy.
Roswell Park Cancer Institute (RPCI) is a worldwide leader in usage of PDT for multiple types of cancers. ${ }^{13}$ The U. S. Food and Drug Administration (FDA) have approved the use of Photofrin for lung cancer as a photosensitizer. Photosan-3, an oligomer of hematoporphyrin, which has been approved by European Union but not by the FDA, has been well documented for its use in photodynamic treatment. ${ }^{14}$ There are several potential benefits of PDT over standard treatments. The primary attraction is the absence of scarring as connective tissue is left intact, including collagen. ${ }^{15}$ The sensitivity of photosensitizers depends on the wavelength of light and the various applications of PDT. The selectivity of treatment imparted is decided by a combination of factors, including accumulation of photosensitizers by the target lesion and targeted application by activating visible or near-infrared (NIR) light. ${ }^{15}$ Marmo et al referred porphyrins and related tetrapyrrole derivatives as an emerging new photosensitizer for tumors of lung, esophagus, skin, and of age-related macular degeneration. ${ }^{16}$ Magnetic resonance imaging (MRI) can be used to evaluate treatment efficacy of PDT in cancer treatment automatically instead of histopathologic assessment. ${ }^{17}$

PDT has several potential benefits compared with standard treatments. The most important criteria for PDT include the choice of photosensitizer and the wavelength of light. Photosensitivity of the cutaneous layer is the most prevalent adverse side effect of systemically administered photosensitizers. The duration of photosensitivity varies from a few days to weeks. Local toxicity depends on the therapy region. PDT is primarily used for superficial tumors and conditions that are not malignant. Since its initial usage in $1700 \mathrm{BC}$, when a glowing tip of a fire drill was used for breast cancer treatment, the use of heat became one of the main techniques for tumor treatment. In PDT, a wavelength spectrum, ranging from radio waves to microwaves and ultrasound waves have been used to induce mild heating in a particular target region called hyperthermia. ${ }^{18,19}$

\section{Mechanism of Photodynamic Therapy}

Light sources used in PDT include coherent light sources such as lasers and noncoherent light sources, such as xenon lamp, argon-pumped laser light-emitting diode (LED), as well as fluorescent lamps. Due to the disadvantages in achieving deep-seated tissues, the effectiveness of PDT is still unexplored. There are mainly two kinds of mechanisms in PDT. In type- 1 mechanism, the toxicity to cancer cells is immediate, caused by the free radicals, whereas in type 2 , the toxicity is due to the conversion of oxygen into singlet oxygen. Some 


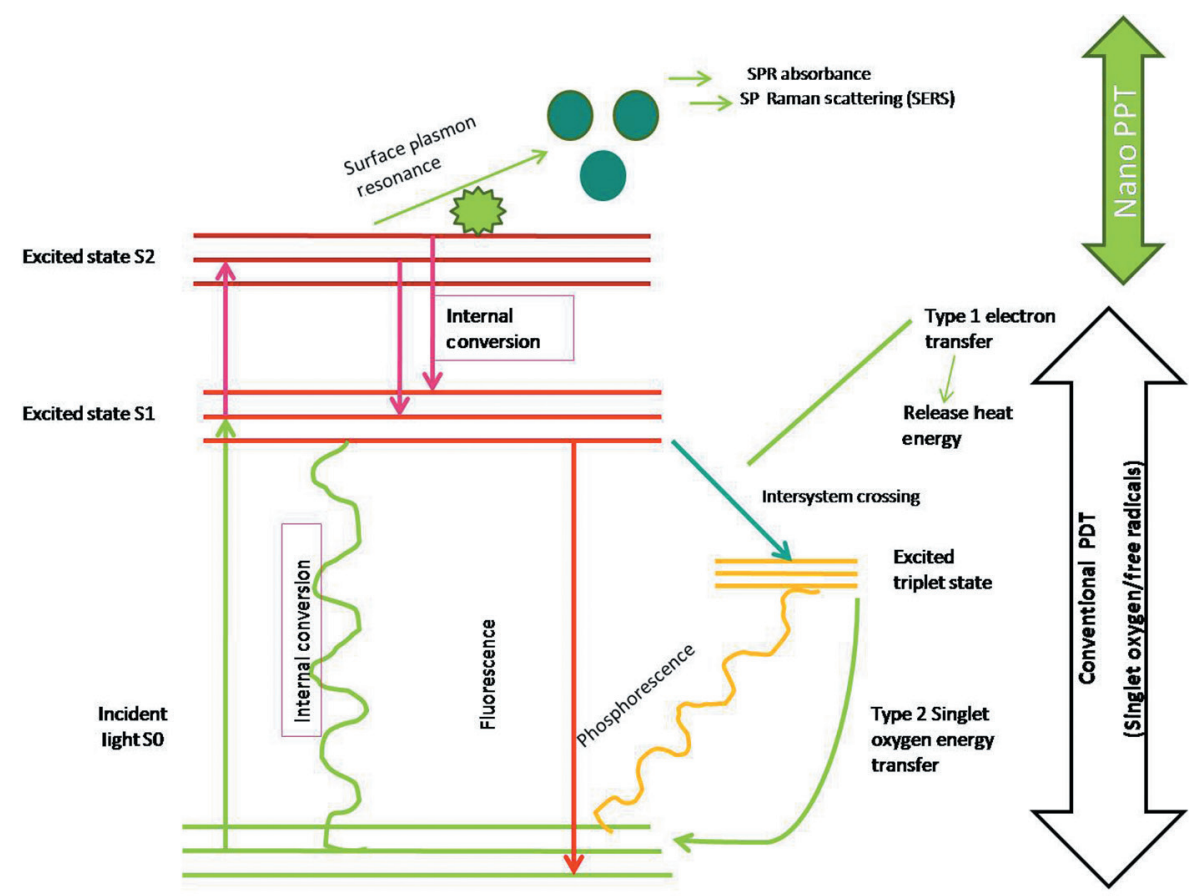

Fig. 3 Modified Jablonski's diagram showing photodynamic therapy mechanism for type I, type II, and nanomaterials. PDT, photodynamic therapy; SPR, surface plasmon resonance.

of the popular photodynamic agents or photosensitizers including hypericin, protoporphyrin, texaphyrin lutetium, and fluorescein halogenated dyes aid in type- 2 pathway where cytotoxic species are generated in the presence of oxygen. The photochemical studies revealed that the membrane protein of the anion channel is the primary target for singlet oxygen $\left({ }^{1} \mathrm{O}_{2}\right)^{19}$ (- Fig. 3 ).

The optimal characteristics of photosensitizers are as follows: constant composition, ease of manufacturing in pure form, nontoxicity in the lack of light, powerful absorption in the visible or IR region, exhibiting target specificity (particularly tumor cell), easy and rapid body clearance, good water solubility (for body fluid compatibility), high triple quantity yield, possession of minimal self-aggregation, the ability to affect tumor in various physiological conditions, and the ability to be photo stable (no photo bleaching). Generally, the existing photosensitizers do not possess all the characteristics of an ideal photosensitizer. ${ }^{20-24}$ The search for an ideal photosensitizer has led to the idea of using nanoparticles as photosensitizers in phototherapy.

\section{Potential of Nanophotonics in Cancer Theranostics}

Nanotechnology refers to a branch of applied science and technology where the concept is the control of matter at the atomic and molecular scale, usually 1 to $100 \mathrm{~nm}$ and to develop products within that size range for a wide range of applications. ${ }^{25,26}$ The classical rules of physics and chemistry do not apply readily in nanotechnology for two main reasons. First, very small particles from their larger cousins can have very different electronic features. Second, the surface-to-quantity ratio becomes much higher and the features of a material change in unexpected ways, as the surface atoms are generally most reactive..$^{26,27}$ The word "nanotech" is widely described as nanometer scale (one billionth of a meter) systems or devices. There is a widespread interest in their use in biomedical systems for diagnosis, imaging, and treatment due to the wide accessibility of nanostructures with greatly regulated optical properties in the nanometer size range..$^{27}$ The capacity to manipulate these nanoparticles' physical, chemical, and binding characteristics enables scientists to design and use the nanoparticles rationally for drug delivery, as image contrast agents and for diagnostic purposes. Nanomaterials' novel characteristics give the capacity to react with biomolecules in new ways. When the size of a material is reduced to the length scale of the nanometer (which is the length scale of the electronic movement that determines the properties of the material), the electronics of the material and therefore the chemical properties change tremendously. ${ }^{28}$

Previously, we described how different kinds of nanoparticles modulate the mechanisms of photonic-mediated cancer therapy and how the changes in the wavelength of light affect the interaction with the nanopartices. ${ }^{29}$ An apparent benefit of nanotechnology refers to biological systems with the capacity to regulate the size of the resulting particles and devices. These nanoparticles have comparable size to many common biomolecules, such as proteins and DNA, thus offering excellent opportunities for the integration of nanotechnology into biotechnology and medicine. The dimensions of the nanoparticles are smaller than the human cells and are comparable to organelles. Biological macromolecules, such as enzymes and receptors, are nanometer in size; hemoglobin is 
around $5 \mathrm{~nm}$ in diameter, while the lipid bilayer surrounding cells is approximately 6-nm thick. Nanoparticles can also cross certain obstacles such as blood-brain barrier, which is usually not the case in other forms of therapeutic and imaging agents. ${ }^{30-32}$ For instance, objects less than $12 \mathrm{~nm}$ in diameter may cross the blood-brain barrier, and objects of $30 \mathrm{~nm}$ or less can be endocytosed by cells. Nanoparticles below $2 \mathrm{~nm}$ can readily pass through the walls of the blood vessels ( - Fig. 4) ${ }^{33-37}$

Nanoparticles are widely classified as metal nanoparticles and semiconductor nanoparticles. In metals, the surface characteristics become dominant and offer unique property to nanoparticles. The consistent collective oscillation of electrons in the conduction band of noble metals induces big surface electrical fields that significantly improve the radiative characteristics of gold and silver nanoparticles when interacting with resonant electromagnetic radiation. This makes the absorption crosssection of these nanoparticles magnitude orders greater than the strongest absorbing molecules and the dispersed light becomes magnitude orders greater than the organic color fluorescence. ${ }^{29}$ These distinctive characteristics provide excellent potential for the use of nanoparticles in many applications such as biochemical sensors, imaging, medical therapy, and catalysis. Applications in drug delivery, cancer cell diagnosis, and treatment are some of the actively studied areas that included quantum dots and gold nanoparticles for cancer imaging and gold nanoparticles for phototherapy as well.

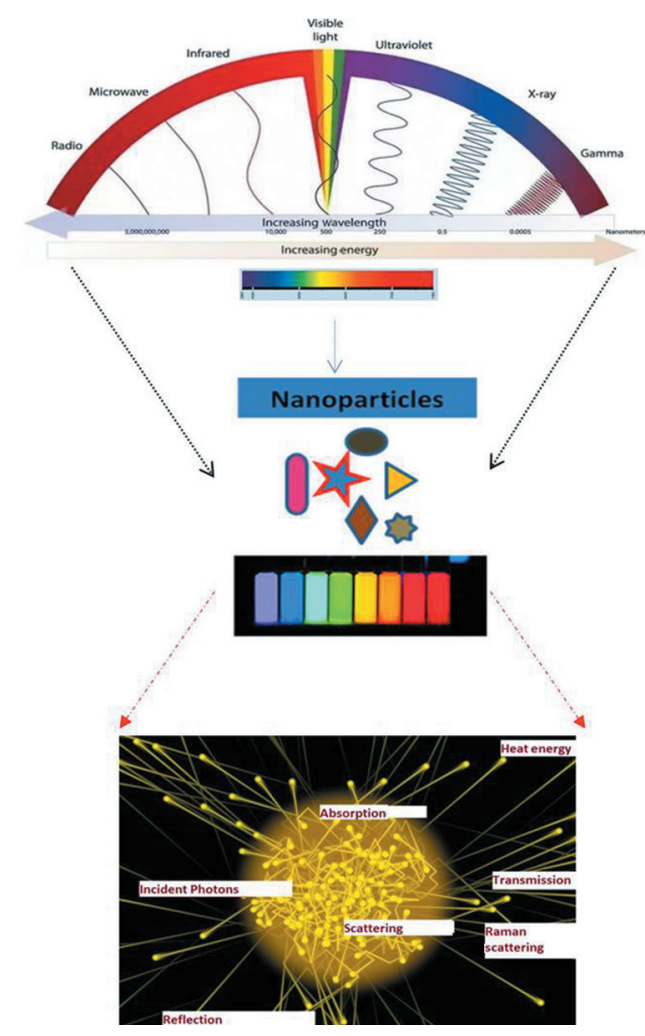

Fig. 4 Potential of nanoparticles photonics interaction.
Normal tissues are frequently interspersed with narrow, capillary vessel walls with 9-nm pores and rarely 50-nm pores. ${ }^{38}$ In addition, these discontinuous capillary walls do not have a basal lamina for easy penetration of particles less than $100 \mathrm{~nm}$. Interestingly, there is no lymphatic system for tumor tissues to eliminate lipophilic and polymeric materials. ${ }^{38}$ Tumors therefore display an improved impact of enhanced permeation and retention (EPR effect) for particles of 50 to $100 \mathrm{~nm}$. Therefore, they cannot be readily removed once the particles enter the tumor tissues. ${ }^{39}$ Nanoparticles which conjugate to appropriate tumor avid biomolecules, with mean sizes in the range of 30 to $50 \mathrm{~nm}$, are ideal for targeting tumors for imaging and therapy purposes. The cell cytoplasm permits nanoparticles which are smaller than $50 \mathrm{~nm}$ and nanoparticles which are smaller than $20 \mathrm{~nm}$ can pass through the vasculature in blood vessels. Chithrani et al investigated the impact of gold nanoparticle (GNP) size, concentration and radiation energy for in vitro radio sensitization of HeLa cells. The same group further reported that $50-\mathrm{nm}$ GNPs are taken up in large numbers than 14- or 74-nm GNPs. ${ }^{40}$ Nanoparticles are reported to overcome the drug resistance and can reduce exocytosis by drug-resistant cells. ${ }^{39}$ Gold spheres of $60 \mathrm{~nm}$ diameter have been used as Surface Enhanced Raman Spectroscopy (SERS) substrates for targeted tumor detection in living mouse. ${ }^{41}$ To monitor the amount of glucose in a living rat, Anker et al created an implantable silver-based SERS sensor. ${ }^{42}$

To efficiently create hybrid nanoparticles for cancer diagnosis and therapy, it is important to understand the distinction between ordinary and cancerous tissue. This can be overcome by nanoparticles as their size and shape depend on the structure and can be optimized for accumulation in tumors. This can also decrease the dramatic side effects induced to healthy tissue, and, at the same time, can enhance their efficacy against cancer cells. The nanoparticle can be easily taken up by cells and recent studies have shown that this can reduce exocytosis by drug-resistant cells. Further, it may be possible to engineer the intracellular distribution of a payload to increase toxicity to the nucleus. Nanotechnology will have a major effect on our cancer detection and treatment capacity leading to decrease in cancer deaths. Molecular biologists work to unravel the molecular networks of the disease. This allows the discovery of nanomaterial diagnostic and therapeutic objectives. To take advantage of this potential, knowledge of the effects of nanomaterial design in in vitro and in vivo conditions is critical which are briefed below:

- The nanoparticles interacts with biomolecules on the surface and within the cell, and the main benefit is that nanoparticles are intact against the molecules' biochemical characteristics. Nanoscale device building and characterization may assist in understanding carcinogenesis.

- Nanoparticles are so versatile that they have a broad optically active bandwidth of the complete spectrum of electromagnetic radiation such as from radio waves to gamma rays. Nanoparticles of different sizes and shapes 
could interact with a full range of photonic spectra including visible range that produces all types of photonic reactions.

- When the size of a material is lowered to the length scale of the nanometer, there is a tremendous change in its electronics and, therefore, chemical properties. In cancer research, metal nanoparticles has been discovered to be advantageous over other sensitizers. In metals, the surface electrons excited by nonionizing radiation convert the light energy into various possible energy such as heat energy, fluorescence or shock waves, and emits the energy to the surrounding areas. The surface electron becomes dominant in metal nanoparticles and gives new properties to nanoparticles. Connor et al have shown that, despite being taken into the cells, gold nanoparticles are inherently nontoxic to human cells..$^{43}$ Some precursors used in the production of nanoparticles, however, may be poisonous. The reported findings were important to control the toxicity of gold nanoparticles by using nontoxic reagents to create them.

- For ophthalmic disease, PDT is presently in exercise. Increasing a cell's temperature results in hyperthermia, coagulation, thermal ablation, vaporization, carbonization, apoptosis, and necrosis. In contrast to the normal temperature $\left(37^{\circ} \mathrm{C}\right)$ of a biological system, a temperature in the range of 45 to $47^{\circ} \mathrm{C}$ is regarded as hyperthermia which can initiate cell apoptosis. The rise in cell temperature up to $60^{\circ} \mathrm{C}$ leads to protein coagulation, followed by macrophage engulfing. A temperature of $100^{\circ} \mathrm{C}$ leads to vaporization, resulting in $80 \%$ boiling of the water content and a temperature of $200^{\circ} \mathrm{C}$ causes cell carbonization.

\section{Future Scope of PDT}

The fast-growing field of nanotechnology offers the chance for interdisciplinary studies. An apparent benefit of nanotechnology is the size of the particles which regulated in such a way that it can be effectively used in biological systems for enhanced penetration and localization. Materials in the nanoscale are smaller than human cells and are comparable to organelles within a cell. In addition, nanoparticles can cross certain obstacles, making it an effective therapeutic and diagnostic tool. By choosing the correct size and shape one can generate nanoparticles, which can be optimized for accumulation in tumors. This can also decrease the dramatic side effects induced to healthy tissue. The shallow penetration of light photons is the biggest challenge of PDT, limiting the usage of PDT only to skin and surface lesions. The combination of nanoparticles with PDT can greatly enhance the efficacy of the treatment. Moreover, sensitizers can be coupled with specific biomarkers for individual patients with cancer which in turn can lead to efficient precision medicine. Such an approach would spare the risk of radiation or emergence of resistance, which are usually associated with radio and chemotherapy. The sensitizer's accurate binding with tumor and treatments using a feeble light source can cure cancer without harming the surrounding environment. Instead of recommending as palliative medicine, PDT and plasmonic phototherapy have the potential to become a primary treatment strategy. The photosensitizers and light source are generally harmless to humans and only in combination create therapeutic reaction. Since radiation induced side effects are not seen in visible and infrared light sources, targeted therapy could be easily achieved with nano-PDT. Hence, PDT opens up a promising field for clinical implementation in precision medicine.

\section{Conflict of Interest}

None declared.

\section{References}

1 Moan J, Peng Q. An outline of the hundred-year history of PDT. Anticancer Res 2003;23(5A):3591-3600

2 Sellera FP, Nascimento CL, Ribeiro MS, eds, Photodynamic Therapy in Veterinary Medicine: From Basics to Clinical Practice. Switzerland; Springer International Publishing; 2016

3 Moan J, Peng Q. An outline of the history of PDT. Photodynam Therap 2003;1-18; doi 10.1039/9781847551658-00001

4 Benov L. Photodynamic therapy: current status and future directions. Med Princ Pract 2015;24(Suppl 1):14-28

5 Akimoto J, Fukami S, Ichikawa M, Mohamed A, Kohno M. Intraoperative photodiagnosis for malignant glioma using photosensitizer talaporfin sodium. Front Surg 2019;6:12

6 Agostinis P, Berg K, Cengel KA, et al. Photodynamic therapy of cancer: an update. CA Cancer J Clin 2011;61:4250-4281

7 Cengel KA, Simone CB II, Glatstein E. PDT: what's past is prologue. Cancer Res 2016;76(9):2497-2499

8 Ackroyd R, Kelty C, Brown N, Reed M. The history of photodetection and photodynamic therapy. Photochem Photobiol 2001;74(5):656-669

9 Kessel D. Introduction to photodynamic therapy. Available at: http://photobiology.info/Kessel.html. Accessed march 27, 2020

10 Evensen JF. The use of porphyrins and non-ionizing radiation for treatment of cancer. Acta Oncol 1995;34(8):1103-1110

11 Dolmans DE, Fukumura D, Jain RK. Photodynamic therapy for cancer. Nat Rev Cancer 2003;3(5):380-387

12 Wiedmann MW, Caca K. General principles of photodynamic therapy (PDT) and gastrointestinal applications. Curr Pharm Biotechnol 2004;5(4):397-408

13 Mayor PC, Lele S. Photodynamic therapy in gynecologic malignancies: a review of the roswell park cancer institute experience. Cancers (Basel) 2016;8(10):E88

14 Photodynamic Therapy. Available at: https://www. aetnabetterhealth.com/pennsylvania/assets/pdf/provider/ guidelines/medical/Medical/Photodynamic-Therapy.pdf. Accessed April 12, 2020

15 Shackley DC, Whitehurst C, Clarke NW, Betts C, Moore JV. Photodynamic therapy. J R Soc Med 1999;92(11):562-565

16 da Silva NS Costa MM, Soares CP. Photodynamic therapy: porphyrins and phthalocyanines as photosensitizers. Aust J Chem 2008;61:741-754

17 Schreurs TJL, Jacobs I, Nicolay K, Prompers JJ, Strijkers GJ. Detection of treatment success after photodynamic therapy using dynamic contrast-enhanced magnetic resonance imaging. Theranostics 2017;7(19):4643-4657

18 Henderson BW, Waldow SM, Potter WR, Dougherty TJ Interaction of photodynamic therapy and hyperthermia: tumor response and cell survival studies after treatment of mice in vivo. Cancer Res 1985;45(12, Pt 1) :6071-6077

19 Celli JP, Spring BQ Rizvi I, et al. Imaging and photodynamic therapy: mechanisms, monitoring, and optimization. Chem Rev 2010;110(5):2795-2838 
20 Kudinova NV, Berezov TT. Photodynamic therapy of cancer: search for ideal photosensitizer. Biochemistry Supplement Series B: Biomedical Chemistry.T.T.Biochem. Moscow Suppl. Ser. B. Springer International Publishing, Switzerland 2010;4(1):95-103

21 Allison RR, Downie GH, Cuenca R, Hu XH, Childs CJ, Sibata CH. Photosensitizers in clinical PDT. Photodiagn Photodyn Ther 2004;1(1):27-42

22 Rishi P, Agarwal V. Current role of photodynamic therapy in ophthalmic practice. Sci J Med \& Vis Res Foun 2015;33:2

23 Mahalingam SM, Ordaz JD, Low PS. Targeting of a photosensitizer to the mitochondrion enhances the potency of photodynamic therapy. ACS Omega 2018;3(6):6066-6074

24 Wang P, Tang H, Zhang P. Plasmonic nanoparticle-based hybrid photosensitizers with broadened excitation profile for photodynamic therapy of cancer cells. Sci Rep 2016;6:34981

25 Banerjee HN, Verma M. Application of nanotechnology in cancer. Technol Cancer Res Treat 2008;7(2):149-154

26 Ghatole S, Dahikar PB, Rokde K. Study of biosensors using nanotechnology. Int J Adv Sci Eng Technol 2015;(1);155-157; ISSN:2321-9009

27 Jeevanandam J, Barhoum A, Chan YS, Dufresne A, Danquah MK. Review on nanoparticles and nanostructured materials: history, sources, toxicity and regulations. Beilstein J Nanotechnol 2018;9:1050-1074

28 Roduner E. Size matters: why nanomaterials are different. Chem Soc Rev 2006;35(7):583-592

29 Poorani G, Rao AP, Singaravelu G, Manickam E. Review of laser in nanophotonics - a literature study for cellular mechanism. J Cancer Biol Res 2017;5(1):1094

30 Salata O. Applications of nanoparticles in biology and medicine. J Nanobiotechnology 2004;2(1):3

31 Behzadi S, Serpooshan V, Tao W, et al. Cellular uptake of nanoparticles: journey inside the cell. Chem Soc Rev 2017;46(14):4218-4244

32 Wang EC, Wang AZ. Nanoparticles and their applications in cell and molecular biology. Integr Biol 2014;6(1):9-26
33 Mesquita MQ Dias CJ, Gamelas S, Fardilha M, Neves MGPS, Faustino MAF. An insight on the role of photosensitizer nanocarriers for photodynamic therapy. An Acad Bras Cienc 2018;90(1, Suppl 2):1101-1130

34 Popp J, Tuchin VV, Chiou A, Heinemann S. Handbook of Biophotonics: Photonics for Health Care. Weinheim, Germany: Wiley-VCH Verlag and Co; 2012: 321-336

35 Abrahamse H, Hamblin MR. New photosensitizers for photodynamic therapy. Biochem J 2016;473(4):347-364

36 Calderhead RG, Tanaka Y, Photobiological Basics and Clinical Indications of Phototherapy for Skin Rejuvenation. Photomedicine - Advances in Clinical Practice. London, United Kingdom: Intech open; 2017:215-252

37 Al-Omari S. Toward a molecular understanding of the photosensitizer-copper interaction for tumor destruction. Biophys Rev 2013;5(4):305-311

38 Shahbazi M-A, Herranz B, Santos HA. Nanostructured porous Si-based nanoparticles for targeted drug delivery. Biomatter 2012;2(4):296-312

39 Golombek SK, May JN, Theek B, et al. Tumor targeting via EPR: Strategies to enhance patient responses. Adv Drug Deliv Rev 2018;130:17-38

40 Chithrani BD, Ghazani AA, Chan WC. Determining the size and shape dependence of gold nanoparticle uptake into mammalian cells. Nano Lett 2006;6(4):662-668

41 Li Y, Wei Q, Ma F, Li X, Liu F, Zhou M. Surface-enhanced Raman nanoparticles for tumor theranostics applications. Acta Pharm Sin B 2018;8(3):349-359

42 Anker JN, Hall WP, Lyandres O. Shah NC, Zhao J, Van Duyne RP. Biosensing with plasmonic nanosensors. Nat Mater 2008;7(6):442-453

43 Connor EE, Mwamuka J, Gole A, Murphy CJ, Wyatt MD. Gold nanoparticles are taken up by human cells but do not cause acute cytotoxicity. Small 2005;1(3):325-327 